\title{
Coadministration of Chemokine Receptor Antagonists with Morphine Potentiates Morphine's Analgesic Effect on Incisional Pain in Rats
}

\author{
Saadet Inan, Toby K. Eisenstein, Mia N. Watson, Menahem Doura, Joseph J. Meissler, \\ Christopher S. Tallarida, Xiaohong Chen, Ellen B. Geller, Scott M. Rawls, Alan Cowan, \\ and Martin W. Adler \\ Center for Substance Abuse Research, Lewis Katz School of Medicine at Temple University, Philadelphia, Pennsylvania \\ Received August 14, 2018; accepted September 21, 2018
}

\begin{abstract}
Crossdesensitization between opioid and chemokine receptors and involvement of chemokines in pain modulation are well established. We investigated if coadministration of chemokine receptor antagonists (CRAs) with morphine would enhance the analgesic potency of morphine on incisional pain in rats. Animals underwent incisional surgery on the left hind paw and pain responses were evaluated using von Frey filaments at various time points postsurgery between 15 and 360 minutes and daily between 24 and 72 hours. Dose-response curves for morphine, maraviroc (a CCR5 antagonist), and AMD3100 (a CXCR4 antagonist) alone were established. While morphine significantly reduced pain in a time- and dose-dependent manner, maraviroc and AMD3100 had no effect by themselves. Coadministration of either maraviroc or AMD3100 with morphine significantly increased morphine's analgesic effect on
\end{abstract}

incisional pain, shifting the dose-response curve to the left 2.3and 1.8-fold, respectively. Coadministration of both CRAs with morphine significantly shifted further the morphine doseresponse curve to the left 3.3-fold. The effect of treatments on mRNA levels in the draining popliteal lymph node for a panel of chemokines and cytokines showed that message for many of these mediators was upregulated by the incision, and the combination of morphine with the CRAs markedly downregulated them. The data show that combining morphine with CRAs potentiates morphine's analgesic effect on incisional pain. Thus, the same analgesic effect of morphine alone can be achieved with lower doses of morphine when combined with CRAs. Using morphine in lower doses could reduce unwanted side effects and possibly block development of tolerance and dependence.

\section{Introduction}

Crosstalk between opioid and chemokine receptors via heterologous desensitization is well established (Grimm et al., 1998; Ali et al., 1999; Zhang et al., 2003, 2004; Chen et al., 2004; Heinisch et al., 2011). Opioid receptors are expressed on macrophages, monocytes, and $\mathrm{T}$ and $\mathrm{B}$ lymphocytes, and their activation with an agonist induces changes in some chemokine levels and chemokine receptor expression (Chuang et al., 1995; Wick et al., 1996; Grimm et al., 1998; Bidlack, 2000; Wetzel et al., 2000; Guo et al., 2002; Mahajan et al., 2002; Happel et al., 2008). It has been shown that met-enkephalin and morphine can inhibit chemotaxis of human neutrophils and peripheral blood mononuclear cells to several chemokines including CXCL1/IL-8, CCL5/RANTES, and CCL2/MCP-1 via phosphorylation of the

This work was supported by the Department of Defense and U.S. Army [Grant W81XWH-15-1-0252]; and the National Institutes of Health National Institute on Drug Abuse [Grant P30 DA013429].

This work was presented, in part, at the International Narcotics Research Conference, 2017, Chicago, IL and the Mid-Atlantic Pharmacology Society, 2017, Philadelphia, PA.

https://doi.org/10.1124/jpet.118.252890. intracellular tails of the chemokine receptors (Grimm et al., 1998). In the central nervous system, chemokine receptors are expressed on microglia, astrocytes, oligodendrocytes, and neurons. They play a role in brain development, angiogenesis, and development of inflammatory as well as degenerative conditions in the central nervous system (Kim and Broxmeyer, 1999; Ragozzino, 2002; Tran and Miller, 2003). Studies using different rodent models show that chemokines play a role in pain (Tanaka et al., 2004; Dansereau et al., 2008; Peters and Eisenach, 2010; Lee et al., 2013). Central or peripheral administration of chemokines enhances pain perception (Szabo et al., 2002; Zhang et al., 2005; Benamar et al., 2008). Furthermore, their central administration decreases the analgesic effect of opioids, providing evidence that heterologous desensitization between opioid and chemokine receptors can occur in the central nervous system as well (Szabo et al., 2002; Chen et al., 2007a,b). Szabo et al. (2002) showed that administering either CXCL12/SDF-1 $\alpha$ or CCL5/RANTES into the periaqueductal gray (PAG) area blocked the analgesic effect of the mu-selective opioid, DAMGO, given 30 minutes later into the PAG area, as measured by cold water tail flick (CWTF) assay. Pretreatment of animals with an antibody against CCL5/RANTES inhibited the hyperalgesia

ABBREVIATIONS: ANOVA, analysis of variance; CL, confidence limit; CRA, chemokine receptor antagonist; CWTF, cold water tail flick; HIV, human immunodeficiency virus; PAG, periaqueductal gray. 
induced by the chemokine in the CWTF assay (Benamar et al., 2008). It has been shown that chemical and inflammatoryinduced pain stimuli are significantly reduced in CCR5 knockout mice compared with wild-type mice (Lee et al., 2013). Furthermore, these researchers also reported that mu-opioid receptor immunoreactivity in the PAG area is higher in CCR5 knockout mice than wild-type mice. Interestingly, gp120, a human immunodeficiency virus (HIV)-1 coat protein that binds to both CCR5 and CXCR4, had a similar desensitizing effect as a chemokine on morphine analgesia in rats using the CWTF assay (Chen et al., 2011). When AMD3100, a CXCR4 receptor antagonist, was given before gp120, it restored the analgesic effect of morphine. Also, electrophysiological studies of PAG neurons showed that gp120 (Chen et al., 2011) and CXCL12 as well as CX3CL1 (Heinisch et al., 2011) inhibit morphine-induced hyperpolarization and reduction of input resistance, which provides evidence for crosstalk between chemokine receptors and mu-opioid receptors on a cellular level. Thus, ligands that bind to chemokine receptors can desensitize mu-opioid receptors and decrease the analgesic effect induced by mu agonists.

In painful syndromes involving inflammation, patients can be refractory to the analgesic effects of morphine. We hypothesized that chemokine receptor antagonists (CRAs), used in combination with morphine, will produce effective analgesia with smaller doses of morphine. Blocking chemokine receptors with a CRA will reduce inflammation, and therefore pain, and will prevent the crossdesensitization induced by chemokine ligands, thereby increasing opioid receptor activity. Such combinations will likely have the added advantage of reducing unwanted side effects of opioids and potential development of tolerance and dependence. Maraviroc (a CCR5 receptor antagonist) and AMD3100 were chosen as the CRAs because both compounds are Food and Drug Administration-approved for human use (maraviroc: an HIV-1 entry inhibitor used as an antiretroviral for HIV-1 treatment; AMD3100: used in lymphoma and multiple myeloma treatment). CRAs were coadministered with morphine in a rat assay of incisional pain (model for clinical postsurgical pain), in which mechanical allodynia is measured.

\section{Materials and Methods}

Animals. Animal care and experimental procedures were approved and performed in accordance with the Institutional Animal Care and Use Committee of Temple University. Male Sprague-Dawley rats (200-240 g; Taconic Biosciences, Germantown, NY) were used in these experiments. They were housed (two in a cage) in a temperaturecontrolled environment with a 12-hour light-dark cycle and were supplied with food and water ad libitum. Rats were acclimated for a week in the central animal facility before surgery and behavioral testing. Two days before the surgery and behavioral testing, rats were acclimated in individual transparent cubicles with a wire mesh floor for 2 days (1 hour each day).

Surgery. On the day of surgery, rats were acclimated for 30 minutes and then their individual baseline values for paw withdrawal threshold were measured using a series of von Frey filaments (North Coast Medical, Inc., Gilroy, CA), with gradually increasing equal logarithmic bending forces (equivalent to $2,4,6,8,10,15,26$, and $60 \mathrm{~g}$ force). The filaments were applied to the plantar side of each hind paw in an ascending manner. Each filament was tested five consecutive times a few seconds apart. A positive response was defined as quick withdrawal or paw flinching after the application of a filament.

Surgery was performed as previously described by Brennan et al. (1996) under isoflurane anesthesia (4\% isoflurane for induction and
$2.5 \%$ isoflurane for maintenance of anesthesia) in aseptic conditions. A 1-cm longitudinal incision (starting $0.5 \mathrm{~cm}$ from the proximal edge of the heel and extending toward the toes) was made with a scalpel through skin and the fascia of the plantar side of the left hind paw. The plantaris muscle was exposed, elevated, and incised longitudinally. Following bleeding control with gentle pressure, the skin was closed with two single interrupted sutures using 5-0 nylon. Animals were brought back to the individual chambers for mechanical allodynia testing. The time when surgeries were completed was designated as time 0 . The animals awoke on the average approximately 5-8 minutes after the end of the surgery, and fully regained consciousness by the first testing time, which was 15 minutes postsurgery. Paw withdrawal thresholds were recorded at time points postsurgery $(15,30,45,60$, 120,240 , and 360 minutes; 24,48 , and 72 hours) by a person blinded to the treatments. Morphine, chemokine receptor antagonists, or their vehicles were injected subcutaneously at $t=25$ minutes postsurgery. Right and left flank areas, as well as behind the neck area (for the third injection), were used to administer compounds or vehicle.

Compounds. Morphine sulfate pentahydrate (NIDA, Bethesda, MD) was dissolved in sterile saline. Maraviroc and AMD3100 were purchased from Sigma-Aldrich (St. Louis, MO). Maraviroc was dissolved in 10\% dimethylsulfoxide (Sigma-Aldrich), and AMD3100 was dissolved in sterile, pyrogen-free water (Aqualite System, Lake Forest, IL).

Experimental Design. Morphine, chemokine receptor antagonists, or their vehicles alone or in combination studies were injected subcutaneously at $t=25$ minutes postsurgery. Dose-response curves for morphine (1-10 mg/kg), maraviroc $(0.5-5 \mathrm{mg} / \mathrm{kg})$, and AMD3100 (1-10 mg/kg) were established. For combination experiments, fixed doses of maraviroc ( 2.5 and $5 \mathrm{mg} / \mathrm{kg}$ ), AMD3100 (2.5 and $6.7 \mathrm{mg} / \mathrm{kg}$ ), or both (maraviroc at $5 \mathrm{mg} / \mathrm{kg}$ and AMD3100 at $6.7 \mathrm{mg} / \mathrm{kg}$ ) were coadministered with different doses of morphine to establish doseresponse curves. In the experiments for coadministration of one CRA with morphine, rats were injected with vehicle plus morphine, a fixed dose of CRA plus morphine, CRA plus saline, or the two vehicles. Morphine doses used for these experiments were $0.25-5 \mathrm{mg} / \mathrm{kg}$. For the coadministration of two CRAs with morphine, animals received one of the following: two vehicles plus morphine, CRAs plus morphine, CRAs plus saline, or three vehicles. Morphine doses used for these experiments were $0.25-2 \mathrm{mg} / \mathrm{kg}$. Percentage of reversal of mechanical allodynia was calculated using the following formula:

$$
\begin{aligned}
& \text { \% reversal of mechanical allodynia } \\
& =[(\text { test threshold })-(\text { predose threshold }) /(\text { baseline threshold }) \\
& \quad-(\text { predose threshold })] \times 100
\end{aligned}
$$

where the test threshold represents the paw pressure response to the filament at the testing time point; the predose threshold represents the response at 15 minutes after surgery and before treatments; and the baseline threshold represents the baseline value before surgery. The percentage of reversal of mechanical allodynia was calculated individually for each rat, and then the mean, S.D., and S.E.M. values were calculated for individual groups. In drug combination studies, the percentage of reversal of mechanical allodynia at 60 minutes was used because morphine's effect was maximal at 60 minutes postsurgery.

Quantitation of mRNA for Immune Mediators. The level of mRNA for a panel of immune mediators was determined using a commercial array, the $\mathrm{RT}_{2}$ Profiler PCR Array for Rat Inflammatory Cytokines and Receptors (Qiagen, Inc., Germantown, MD). The draining popliteal lymph nodes were collected from animals subjected to the same surgery and treatments as shown in Fig. 7, but they were sacrificed at 1 hour postincision. Tissues were processed by the Qiagen RNeasy Microarray Tissue Mini Kit following the manufacturer's protocols. Individual mRNA preparations were quantitated by a Nanodrop 2000 spectrophotometer (Thermo Scientific, Waltham, MA) to determine mRNA concentrations in nanograms per microliter. mRNAs from individual lymph nodes were used to generate cDNAs 
using the $\mathrm{RT}^{2}$ First Strand Kit (Qiagen, Inc.), which were then assayed using the $\mathrm{RT}^{2}$ Profiler PCR Array with the $\mathrm{RT}^{2}$ SYBR Green ROX qPCR Mastermix (Qiagen, Inc.). This array has probes for mRNA for 84 immune mediators, which include 22 chemokines, 20 cytokines, 21 receptors, and 21 other molecules that are involved in the inflammatory response. The polymerase chain reaction array was run on an ABI StepOne Plus RT-PCR thermocycler (Applied Biosystems, Foster City, CA) using the cycling conditions given in the protocol supplied with the $\mathrm{RT}^{2}$ Array. Data were processed by the online GeneGlobe Data Analysis Center (Qiagen, Inc.). The results obtained are expressed as the $\log _{2}$ fold difference in mRNA expression for each mediator in the array of the treated groups compared with the level of mRNA expression in the baseline untreated control.

Statistical Analysis. Data for morphine, maraviroc, and AMD3100 time-course and dose-response curves as well as data on the combination of morphine with each or both CRAs were analyzed using two-way analysis of variance (ANOVA) followed by Tukey's or Sidak's multiple comparisons tests.

To establish the $\mathrm{ED}_{50}$ values and $95 \%$ confidence limits (CLs) for morphine and for the combination of morphine with a single CRA or with both CRAs, data from dose-response curves measuring percentage of reversal of mechanical allodynia at 60 minutes were used and analyzed by nonlinear regression analysis. To compare dose-response curves for morphine alone and in combination with CRAs, the paired Student's $t$ test was used (Tallarida et al., 2014). Data are expressed as mean \pm S.E.M., and $P<0.05$ was accepted as statistically significant. GraphPad Prism version 7, (GraphPad, La Jolla, CA) was used for data analysis. Statistics on the results of the $\mathrm{RT}^{2}$ Profiler Arrays were done by Student's $t$ test, using the online GeneGlobe Data Analysis Center (Qiagen, Inc.).

\section{Results}

Morphine, but Not Maraviroc or AMD3100, Induces Analgesia in a Time- and Dose-Dependent Manner. Figures 1-3 show the time- and dose-dependent percentages of reversal of mechanical allodynia for rats receiving morphine alone, maraviroc alone, or AMD3100 alone, respectively. Baseline (preincision) paw withdrawal thresholds were similar for all rats in the different groups (33.91 $\pm 2.28 \mathrm{~g})$, and they dropped abruptly 15 minutes after the incision in all groups $(3.22 \pm 0.20 \mathrm{~g})$. Time 0 is the time surgery is completed; 15 minutes postsurgery is the time that baseline measurements were taken; and 25 minutes postsurgery is the time when drugs were administered. Morphine demonstrated an analgesic effect in a time- and dose-dependent manner on incisional pain. Morphine at 5 and $10 \mathrm{mg} / \mathrm{kg}$ induced significant analgesia compared with saline $(P<0.001)$. The analgesic effect started at 45 minutes, reached a maximum at 60 minutes, lasted until 120 minutes, and disappeared by 240 minutes postsurgery. Morphine at 1 and $3 \mathrm{mg} / \mathrm{kg}$ did not produce analgesia, as shown in Fig. 1, where two-way ANOVA revealed significant effects of treatment $[F(4,240)=22.91, P<0.0001]$ and time $[F(5,240)=$ $9.08, P<0.0001]$, and a significant interaction $[F(20,240)=1.786$, $P=0.022]$. Paw withdrawal thresholds were also measured at 24 , 48 , and 72 hours, but no analgesia was observed at these time points (data not shown). The $\mathrm{ED}_{50}$ value for morphine was calculated as $2.34 \mathrm{mg} / \mathrm{kg}$ (95\% CL: 1.69-3.17) (Table 1). Neither maraviroc nor AMD3100 had an analgesic effect on incisional pain (Figs. 2 and 3, respectively).

Effect of Coadministration of Maraviroc or AMD3100 with Morphine on Morphine's Analgesic Activity. The following experiments were carried out to test the effect of combining a CRA with morphine. First, fixed doses of maraviroc $(2.5$ or $5 \mathrm{mg} / \mathrm{kg}$ ) were given with different doses

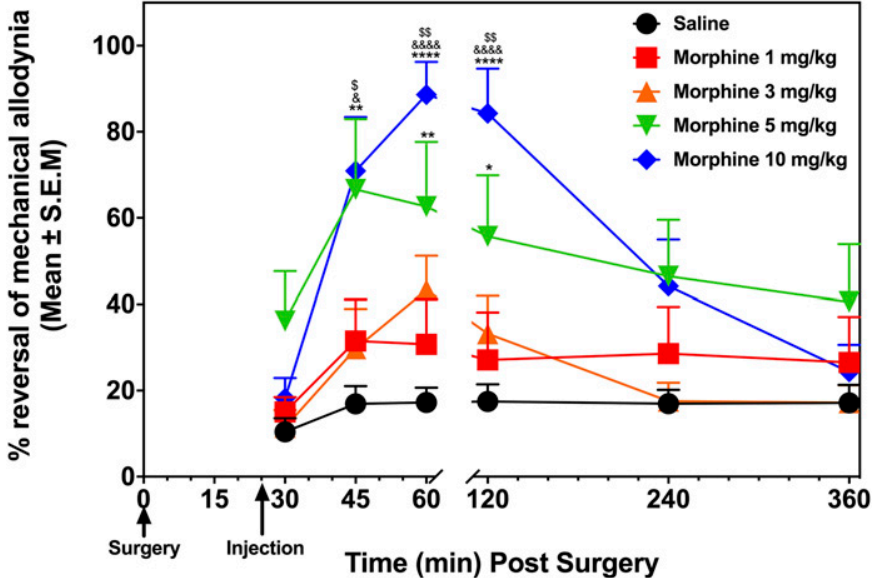

Fig. 1. Morphine time- and dose-response results on incisional pain. After baseline measurements, rats underwent surgery $(t=0)$. At 15 minutes, postsurgery baseline was recorded. Animals were injected with either saline or morphine (subcutaneously) at $t=25$ minutes and paw withdrawal thresholds were measured at $30,60,120,240$, and 360 minutes. Percentage of reversal of mechanical allodynia was calculated. Morphine induced analgesia in a time- and dose-dependent manner. The analgesic effect started at 45 minutes, reached a maximum at 60 minutes, and lasted for 120 minutes postsurgery. Paw withdrawal thresholds also were measured at 24,48 , and 72 hours. No analgesia was observed at these time points (data not shown). Two-way ANOVA followed by Tukey's multiple comparisons test was applied. $* P<0.05$; ** $P<0.01$; *** $P<0.001$; **** $P<0.0001$ compared with saline; ${ }^{\&} P<0.05$; \&\&\&\& $P<0.0001$ compared with $1 \mathrm{mg} / \mathrm{kg}$; ${ }^{\$} P<0.05 ;{ }^{\$ \$} P<0.01$ compared with $3 \mathrm{mg} / \mathrm{kg}(n=8-10)$.

of morphine. Maraviroc at $2.5 \mathrm{mg} / \mathrm{kg}$ was coadministered with morphine at doses ranging from 1 to $5 \mathrm{mg} / \mathrm{kg}$. Since the maximal effect with morphine was established at 60 minutes postsurgery, the percentage of reversal of mechanical allodynia was calculated for all groups at this time point. As seen in Fig. $4 \mathrm{~A}$, coadministration of maraviroc at $2.5 \mathrm{mg} / \mathrm{kg}$ with morphine at $5 \mathrm{mg} / \mathrm{kg}$ significantly increased the analgesic effect compared with $10 \%$ dimethylsulfoxide plus morphine (5 $\mathrm{mg} / \mathrm{kg})$ at $P<0.05$, where two-way ANOVA revealed significant effects of treatment $[F(1,53)=6.746, P=0.012]$ and dose $[F(3,53)=16.36, P<0.0001]$, and a significant interaction $[F(3,53)=3.304, P=0.02]$. While morphine alone at $5 \mathrm{mg} / \mathrm{kg}$ reversed mechanical allodynia by $49.8 \% \pm 13.1 \%$, addition of maraviroc at $2.5 \mathrm{mg} / \mathrm{kg}$ increased this effect to $100 \% \pm 0 \%(P<0.01)$. The $\mathrm{ED}_{50}$ value for the combination of morphine and maraviroc at $2.5 \mathrm{mg} / \mathrm{kg}$ was calculated as $1.95 \mathrm{mg} / \mathrm{kg}$ (95\% CL: 1.08-2.96). In a subsequent experiment, the dose of maraviroc was increased to $5 \mathrm{mg} / \mathrm{kg}$ and used in combination with morphine at doses ranging from 0.5 to $5 \mathrm{mg} / \mathrm{kg}$, where two-way ANOVA revealed significant effects of treatment $[F(1,69)=5.993, P=0.016]$ and dose $[F(4,69)=12.62, P<0.0001]$ but not a significant effect of interaction $[F(4,69)=1.088, P=0.36]$. The $\mathrm{ED}_{50}$ value for this combination was calculated as $1.01 \mathrm{mg} / \mathrm{kg}$ (95\% CL: $0.60-1.56$ ) (Table 1). Coadministration of maraviroc $5 \mathrm{mg} / \mathrm{kg}$ with morphine significantly shifted the morphine-dose response to the left 2.3 -fold ( $P<0.05$, paired Student's $t$ test) (Fig. 7).

Similar experiments were carried out with AMD3100 (2.5 and $6.7 \mathrm{mg} / \mathrm{kg}$ ) with morphine (doses ranging from 1 to 5 and 0.5 to $3 \mathrm{mg} / \mathrm{kg}$, respectively). The results for coadministration of AMD3100 $2.5 \mathrm{mg} / \mathrm{kg}$ with morphine at $5 \mathrm{mg} / \mathrm{kg}$, where two-way ANOVA revealed a significant effect of dose $[F(4,61)=9.776$, 


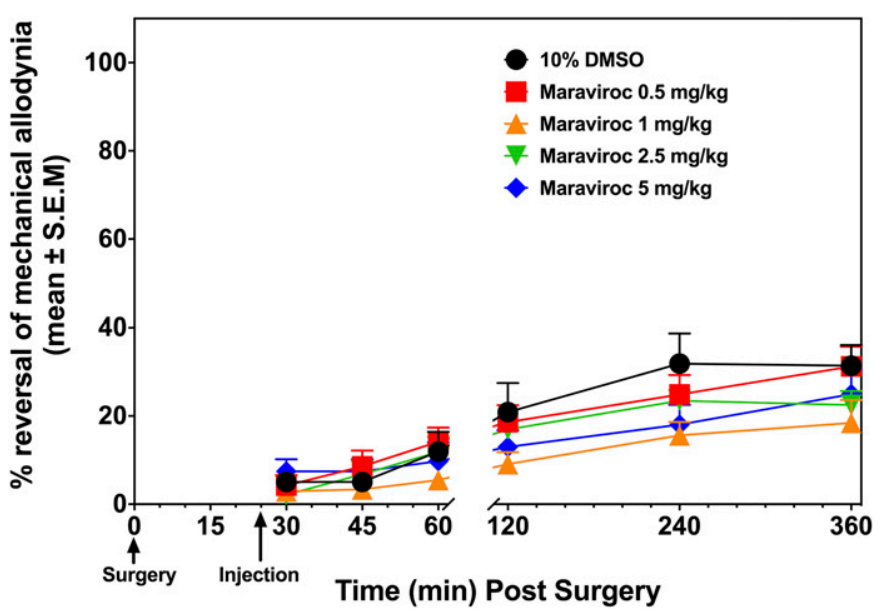

Fig. 2. Maraviroc time- and dose-response results on incisional pain. Determination of baseline values and surgery are the same as in Fig. 1. Animals were then injected with either $10 \%$ dimethylsulfoxide (DMSO) or maraviroc (subcutaneously) at $t=25$ minutes and paw withdrawal thresholds were measured at $30,60,120,240$, and 360 minutes postsurgery. Percentage of reversal of mechanical allodynia was calculated. Two-way ANOVA followed by Tukey's multiple comparisons test revealed no significant differences between doses at any of the time points tested $(n=8-10)$.

$P<0.0001]$ but not of treatment $[F=(1,61)=1.27, P=0.26]$ and interaction $[F=(4,61)=1.88, P=0.12]$, and AMD3100 at $6.7 \mathrm{mg} / \mathrm{kg}$ with morphine at $3 \mathrm{mg} / \mathrm{kg}$, where two-way ANOVA revealed a significant effect of dose $[F(4,69)=20.27$, $P<0.0001]$ but not of treatment $[F(1,69)=3.51, P=0.06]$ and interaction $[F(4,69)=1.66, P=0.16]$, are shown in Figs. 5A and $\mathrm{B}$, respectively. The $\mathrm{ED}_{50}$ values for these combinations were $2.61 \mathrm{mg} / \mathrm{kg}$ (95\% CL: $1.78-3.41)$ and $1.32 \mathrm{mg} / \mathrm{kg}$ (95\% CL: 0.85-1.89), respectively (Table 1 ). Coadministration of AMD3100 $6.7 \mathrm{mg} / \mathrm{kg}$ with morphine significantly shifted the morphine-dose response to the left 1.8 -fold $(P<0.05$, paired Student's $t$ test) (Fig. 7).

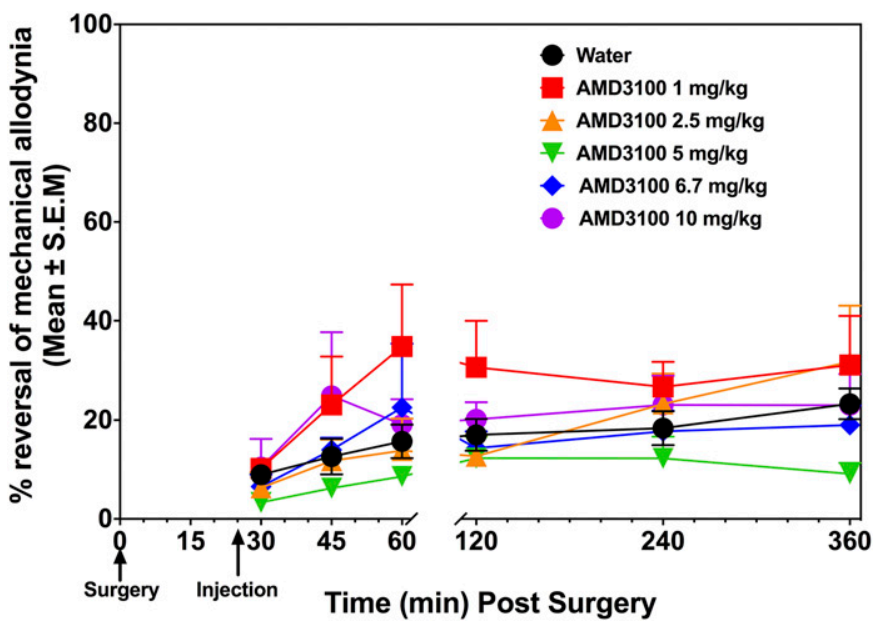

Fig. 3. AMD3100 time- and dose-response results on incisional pain. Determination of baseline values and surgery are the same as in Fig. 1. Animals were injected with either water or AMD 3100 (subcutaneously) at $t=25$ minutes and paw withdrawal thresholds were measured at 30,60 , 120, 240, and 360 minutes postsurgery. Percentage of reversal of mechanical allodynia was calculated. No analgesia was observed with AMD3100. Two-way ANOVA followed by Tukey's multiple comparisons test revealed no significant differences $(n=8-10)$.
TABLE 1

Calculated $\mathrm{ED}_{50}$ values plus $95 \%$ confidence limits for morphine in combination with fixed doses of maraviroc or AMD3100, or a combination with morphine plus maraviroc and AMD3100

\begin{tabular}{lcc}
\hline \multicolumn{1}{c}{ Treatment } & $\begin{array}{c}\text { Calculated ED } \\
\text { Morphine }\end{array}$ & $\begin{array}{c}95 \% \text { Confidence } \\
\text { Interval }\end{array}$ \\
\hline & $m g / k g$ \\
Morphine alone & 2.34 & $1.69-3.17$ \\
Morphine + Maraviroc & 1.01 & $0.60-1.56$ \\
Morphine + AMD3100 & 1.32 & $0.85-1.89$ \\
Morphine + Maraviroc + AMD3100 & 0.71 & $0.42-1.14$ \\
\hline
\end{tabular}

Coadministration of Both Maraviroc and AMD3100 with Morphine Significantly Enhances the Potency of Morphine. Since maraviroc and AMD3100 were each able to increase the analgesic activity of morphine, the effect of a combination of both CRAs with morphine was tested. Empirically, it was decided to test the highest doses of each of the CRAs with doses of morphine that alone gave below $40 \%$ analgesia. As shown in Fig. 6, coadministration of both CRAs with morphine significantly potentiated morphine's analgesic effect, where two-way ANOVA revealed significant effects of treatment $[F(1,76)=17.01, P<0.001]$ and dose $[F(4,76)=$ $8.11, P<0.001]$ and a significant interaction $[F(4,76)=2.67$, $P=0.038$ ]. A significant increase in reversing mechanical allodynia was observed starting with doses of morphine as low as $0.5 \mathrm{mg} / \mathrm{kg}(P<0.05)$. A dose of only $2 \mathrm{mg} / \mathrm{kg}$ of morphine, which is one-fifth of its maximal effective dose, reversed mechanical allodynia by $74.5 \% \pm 11.1 \%$ (Fig. $6, P<0.001$ ). The $\mathrm{ED}_{50}$ value for this combination of two CRAs plus morphine was calculated as $0.71 \mathrm{mg} / \mathrm{kg}$ (95\% CL: 0.42-1.14), which shifted the morphine dose-response 3.3 -fold to the left $(P<0.05)$. Dose-response curves for morphine alone, maraviroc $(5 \mathrm{mg} / \mathrm{kg})$ plus morphine, AMD3100 $(6.7 \mathrm{mg} / \mathrm{kg})$ plus morphine, and maraviroc (5 mg/kg) plus AMD3100 $(6.7 \mathrm{mg} / \mathrm{kg})$ plus morphine are shown in Fig. 7.

Effect of Treatment with Morphine and CRAs on mRNA Levels for Chemokines and Cytokines. Lymph node samples were processed to obtain mRNA as described in Materials and Methods. The objective was to screen a large number of immune mediators to assess how the surgery and treatments with morphine, CRAs, or the combination of morphine plus CRAs altered levels of chemokines and cytokines. Although mRNA levels for 84 analytes were measured by the $\mathrm{RT}^{2}$ Profiler Array, Fig. 8 only presents the results for the ones of interest or with significant changes. It is evident from Fig. 8, A and B, that the incision alone caused a statistically significant increase (starred bars) in mRNAs for several cytokines and chemokines, including CCL2/MCP-1, CCL7/MCP-3, CCL12, CXCL2/MIP-2, IL-1 $\alpha$, IL-1 $\beta$, IL-5, and G-CSF. There was a trend toward increased mRNA levels for CCL20/MIP-3 $\alpha$ and CXCL1. Morphine alone at $2 \mathrm{mg} / \mathrm{kg}$ did not have a strong effect on the mRNA levels of mediators in this panel. The two CRAs, in spite of having little effect on pain, did reduce $\mathrm{mRNA}$ for five of the eight mediators upregulated by the incision to a level that was not significantly above baseline. The combination of the suboptimal dose of morphine plus the two CRAs decreased the mRNA levels of all of the mediators that were significantly upregulated by the incision, such that none of them were significantly different from baseline values. mRNAs for several of the mediators were also more robustly downregulated by the morphine + two CRAs than by the CRAs 
A

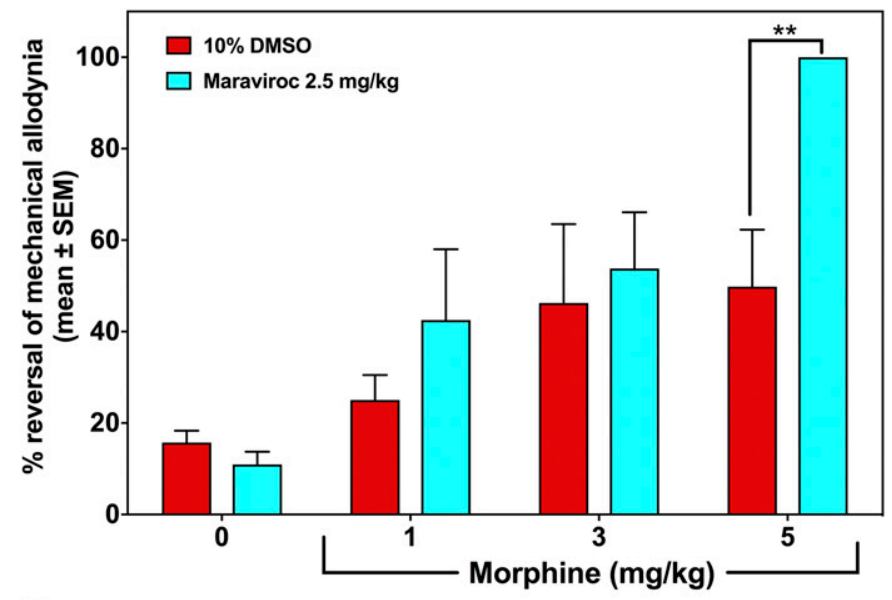

B

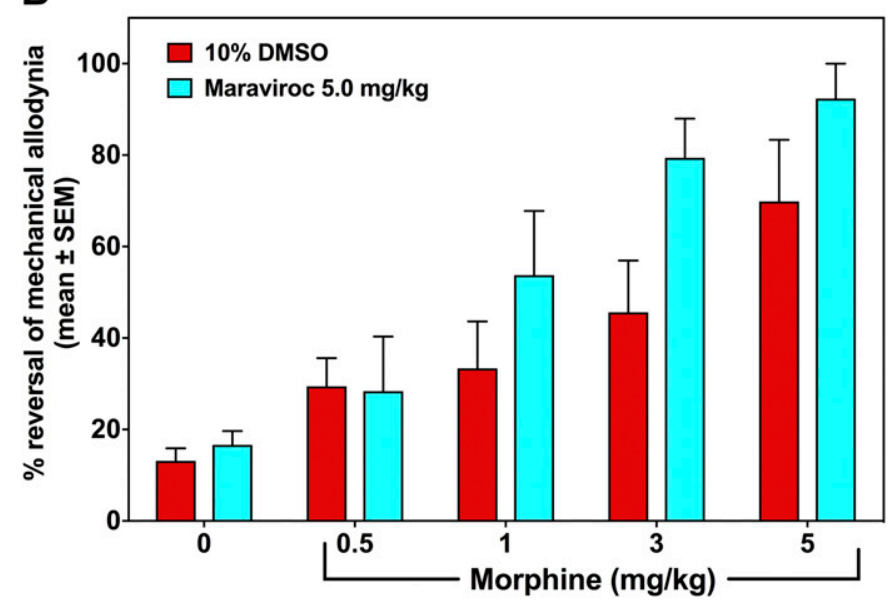

Fig. 4. Effect of the coadministration of maraviroc with morphine on incisional pain. Determination of baseline values and surgery are the same as in Fig. 1. (A) Animals were injected at $t=25$ minutes with $10 \%$ dimethylsulfoxide (DMSO) + morphine (subcutaneously) or maraviroc $(2.5 \mathrm{mg} / \mathrm{kg})+$ morphine (subcutaneously). (B) Animals were injected at $t=25$ minutes with $10 \%$ DMSO + morphine (subcutaneously) or maraviroc $(5 \mathrm{mg} / \mathrm{kg})+$ morphine (subcutaneously). Paw withdrawal thresholds were measured from 30 to 360 minutes. Percentage of reversal of mechanical allodynia was calculated using data at 60 minutes. Coadministration of maraviroc $(2.5 \mathrm{mg} / \mathrm{kg})$ with morphine $(5 \mathrm{mg} / \mathrm{kg})$ significantly increased morphine's analgesic effect (A). Two-way ANOVA followed by Sidak's multiple comparisons test showed $* * P<0.01(n=8-10)$.

without morphine. (Baseline values are not available, but are intrinsic to the assay.) Surprisingly, neither mRNA for CCL5/RANTES nor for CXCL12/SDF-1 $\alpha$ were upregulated by the incision alone, and there was no effect on mRNA for these chemokines in animals receiving the antagonists for their receptors (AMD3100 for CXCR4 and maraviroc for CCR5). Instead, the combination of morphine plus the two CRAs downregulated a broader panel of mRNAs for unrelated proinflammatory chemokines and cytokines.

\section{Discussion}

The present study tested the hypothesis that simultaneous administration of one or two CRAs with a subanalgesic dose of morphine would increase morphine's analgesic potency. It is shown here for the first time that a CRA, having no analgesic effect by itself, when given together with a subanalgesic dose
A
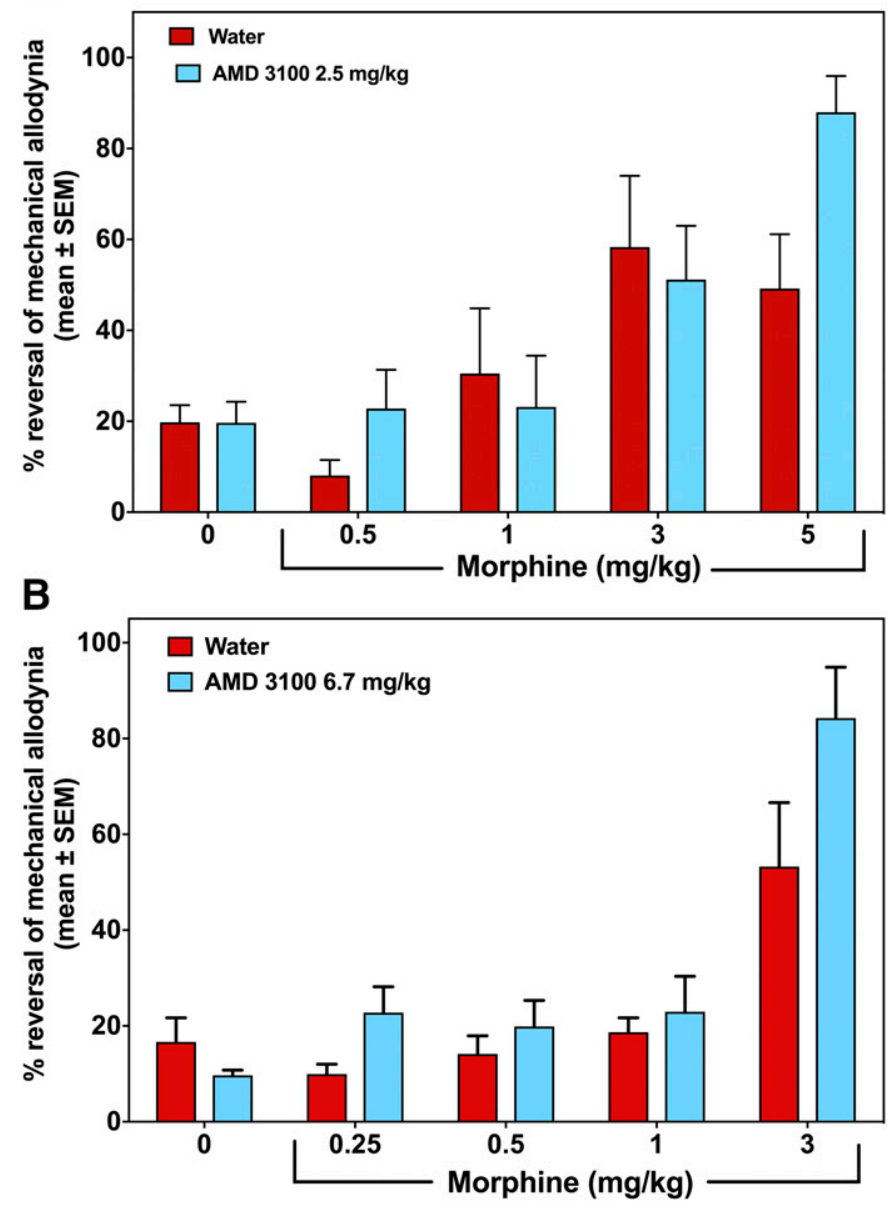

Fig. 5. Effect of coadministration of AMD3100 with morphine on incisional pain. Determination of baseline values and surgery are the same as in Fig. 1. (A) Animals were injected at $t=25$ minutes with water + morphine (subcutaneously) or AMD3100 $(2.5 \mathrm{mg} / \mathrm{kg})+$ morphine (subcutaneously). (B) Animals were injected at $t=25$ minutes with water + morphine (subcutaneously) or AMD3100 $(6.7 \mathrm{mg} / \mathrm{kg})+$ morphine (subcutaneously). Paw withdrawal thresholds were measured at time points between 30 and 360 minutes. Percentage of reversal of mechanical allodynia was calculated using data at 60 minutes. Coadministration of AMD3100 $(2.5 \mathrm{mg} / \mathrm{kg})$ with morphine $(5 \mathrm{mg} / \mathrm{kg})(\mathrm{A})$ and coadministration of AMD3100 $(6.7 \mathrm{mg} / \mathrm{kg})$ with morphine $(3 \mathrm{mg} / \mathrm{kg})(B)$ show a trend to increase analgesia (two-way ANOVA revealed significance only for dose) $(n=8-10)$.

of morphine, modestly enhances analgesia. However, when two CRAs were administered together with morphine, there was a much more robust and significant enhancement in the analgesic effect of morphine on incisional pain. To our knowledge, this is the first report showing that a combination of two CRAs with morphine enhances analgesia. When administered together with morphine, either maraviroc or AMD3100 each separately reduced the $\mathrm{ED}_{50}$ value of morphine from 2.34 to 1.01 and $1.32 \mathrm{mg} / \mathrm{kg}$, respectively. When both CRAs were combined with morphine, they further potentiated and reduced the $\mathrm{ED}_{50}$ value of morphine to $0.71 \mathrm{mg} / \mathrm{kg}$. Compared with morphine alone, this is a 3.3 -fold reduction of the morphine dose. In these experiments, it should be noted that rats were not pretreated. The drugs were administered therapeutically, which simulates the human postsurgery condition, and is a more stringent paradigm than a prophylactic experimental design. Furthermore, this 


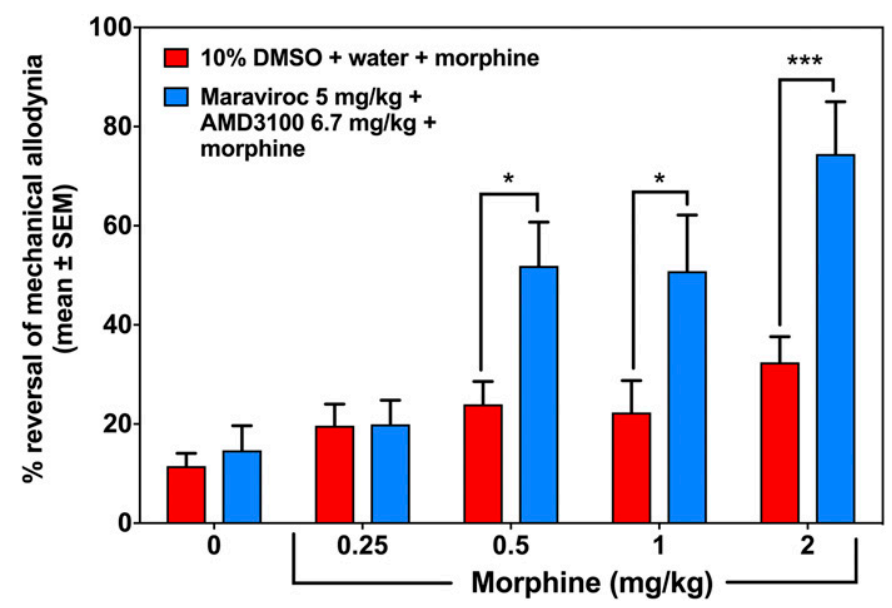

Fig. 6. Effect of coadministration of maraviroc $(5 \mathrm{mg} / \mathrm{kg})$ and AMD3100 $(6.7 \mathrm{mg} / \mathrm{kg})$ with morphine on incisional pain. Determination of baseline values and surgery are the same as in Fig. 1 . At $t=25$ minutes, animals were injected with either $10 \%$ dimethylsulfoxide (DMSO) + water + saline, $10 \%$ DMSO + water + morphine, maraviroc $(5 \mathrm{mg} / \mathrm{kg})+$ AMD3100 $(6.7 \mathrm{mg} / \mathrm{kg})+$ saline, or maraviroc $(5 \mathrm{mg} / \mathrm{kg})+$ AMD3100 $(6.7 \mathrm{mg} / \mathrm{kg})+$ morphine. Percentage of reversal of mechanical allodynia was calculated using data at 60 minutes. Combining two CRAs with subanalgesic doses of morphine $(0.5,1$, and $2 \mathrm{mg} / \mathrm{kg}$ ) significantly increased morphine's analgesic effects. Two-way ANOVA followed by Tukey's multiple comparisons test showed $* P<0.05 ; * * P<0.01(n=6-10)$.

study is the first to test the effects of maraviroc and AMD3100 by themselves, as well as in combination with morphine, on incisional pain. Maraviroc has been studied for neuropathic pain (Kwiatkowski et al., 2016; Piotrowska et al., 2016) and for remifentanil-induced hyperalgesia (Li et al., 2016) in rodents. Kwiatkowski et al. (2016) administered maraviroc intrathecally before chronic constrictive nerve injury, and then continued by intrathecal injection for 7 days. Maraviroc significantly reduced mechanical allodynia by itself, and significantly increased analgesic effects of both morphine and buprenorphine on neuropathic pain. Also, chronic oral administration of a dual CCR2 and CCR5 antagonist, RAP103 , induced analgesia to neuropathic pain in rats induced by chronic, constrictive nerve injury (Padi et al., 2012). Li et al. (2016) also administered maraviroc intrathecally before remifentanil and found that pretreatment with maraviroc inhibited remifentanil-induced thermal and mechanical allodynia. Akgün et al. (2015) showed that intrathecal administration of a synthetized bivalent compound, which has both mu-opioid agonist and CCR5 antagonist activity, was highly potent in preventing inflammatory and neuropathic pain compared with morphine in mice pretreated with lipopolysaccharide. The findings of that study support the present study in that the result was to increase the potency of morphine's analgesic effect when combined with a CCR5 antagonist.

Previously, our laboratories have shown that individual administration of several different chemokines into the PAG area, before morphine administration, blocked the analgesic effect of morphine in the CWTF test (Szabo et al., 2002). There is evidence that opioid receptors and chemokine receptors can form heterodimers (Chen et al., 2004), which lead to heterologous desensitization of the opioid receptors. Furthermore, single neurons in the PAG area express both opioid and chemokine receptors, which would allow for heterodimer formation (Heinisch et al., 2011). The hypothesis to explain the current results, showing that the combination of a

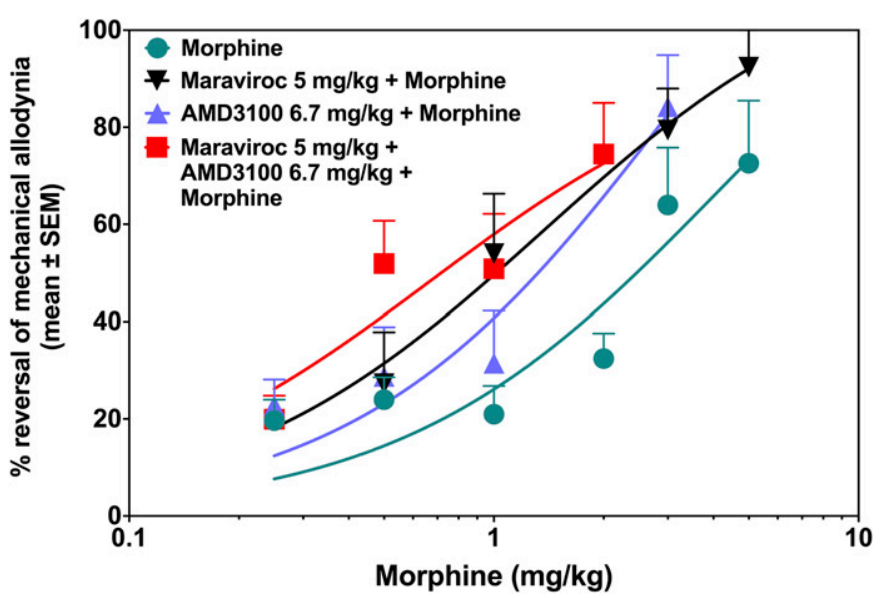

Fig. 7. Combination of morphine with two CRAs shifts the morphine dose-response curve to the left. Percentage of reversal of mechanical allodynia was calculated using data at 60 minutes. The combination of morphine with either AMD3100 or maraviroc individually, as well as the combination of morphine with both CRAs, significantly shifted the morphine dose-response curve to the left. The paired Student's $t$ test showed $* P<0.05(n=6-10)$

chemokine receptor antagonist with morphine can potentiate the activity of morphine, is that the CRA is preventing crossdesensitization of the opioid receptor by chemokines released in the inflammatory response resulting from the incision. It would be consistent with the hypothesis that two CRAs would be superior to a single CRA by preventing crossdesensitization at multiple chemokine receptors. The predicted result would be to increase the availability of additional mu receptors.

The present study is unique in that the maraviroc was administered systemically. Furthermore, the effect on incisional pain was evaluated using the drugs therapeutically rather than prophylactically. Finally, maraviroc increased morphine's analgesic effect on postsurgery pain. The reason why maraviroc by itself was ineffective in the present study may be that maraviroc was not administered chronically; it was given therapeutically after the insult, and the pain induced by the incision may have different mechanisms than neuropathic pain. Additionally, in a clinical study performed in patients with rheumatoid arthritis treated with methotrexate, maraviroc was found ineffective (Fleishaker et al., 2012).

AMD3100 has also been studied for possible analgesic effects in neuropathic pain in rodents. Intrathecal administration of AMD3100 significantly reduced mechanical allodynia induced by sciatic nerve ligation (Luo et al., 2016) and HIV gp120 (Huang et al., 2014). Additionally, systemic administration of AMD3100 improved mechanical allodynia in diabetic neuropathy (Menichella et al., 2014) and following spinal nerve ligation (Xie et al., 2016). In contrast to these reports, in the present study there was no analgesic effect when AMD3100 was given alone. Possible reasons for this discrepancy are that in the current studies the CRA was administered as a single dose, one time, and in a different pain model.

In the present study, changes in chemokine and cytokine mRNA expression induced by incision and the various treatments were studied in popliteal lymph nodes. Several cytokine and chemokine mRNAs, including those for CCL2/MCP-1, CCL7/MCP-3, CCL12, CXCL2/MIP-2, IL-1 $\alpha$, IL-1 $\beta$, IL-5, and 
A

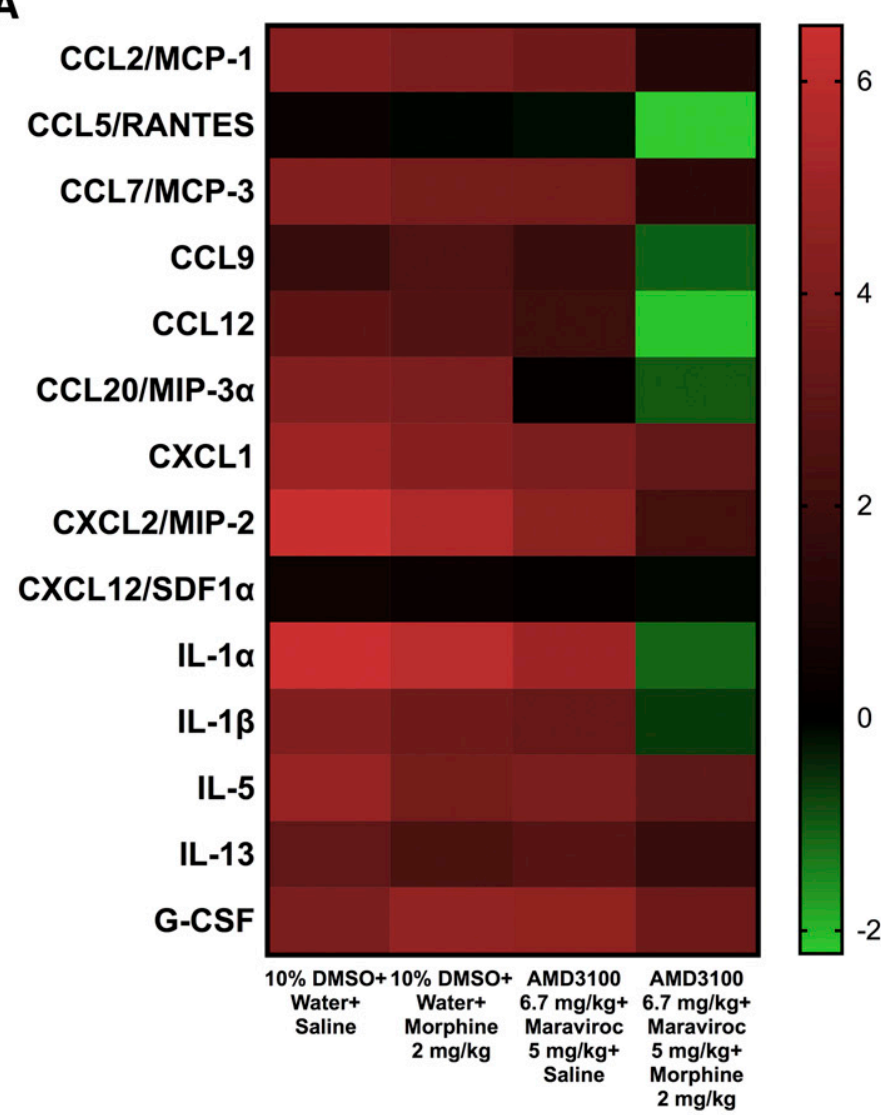

B
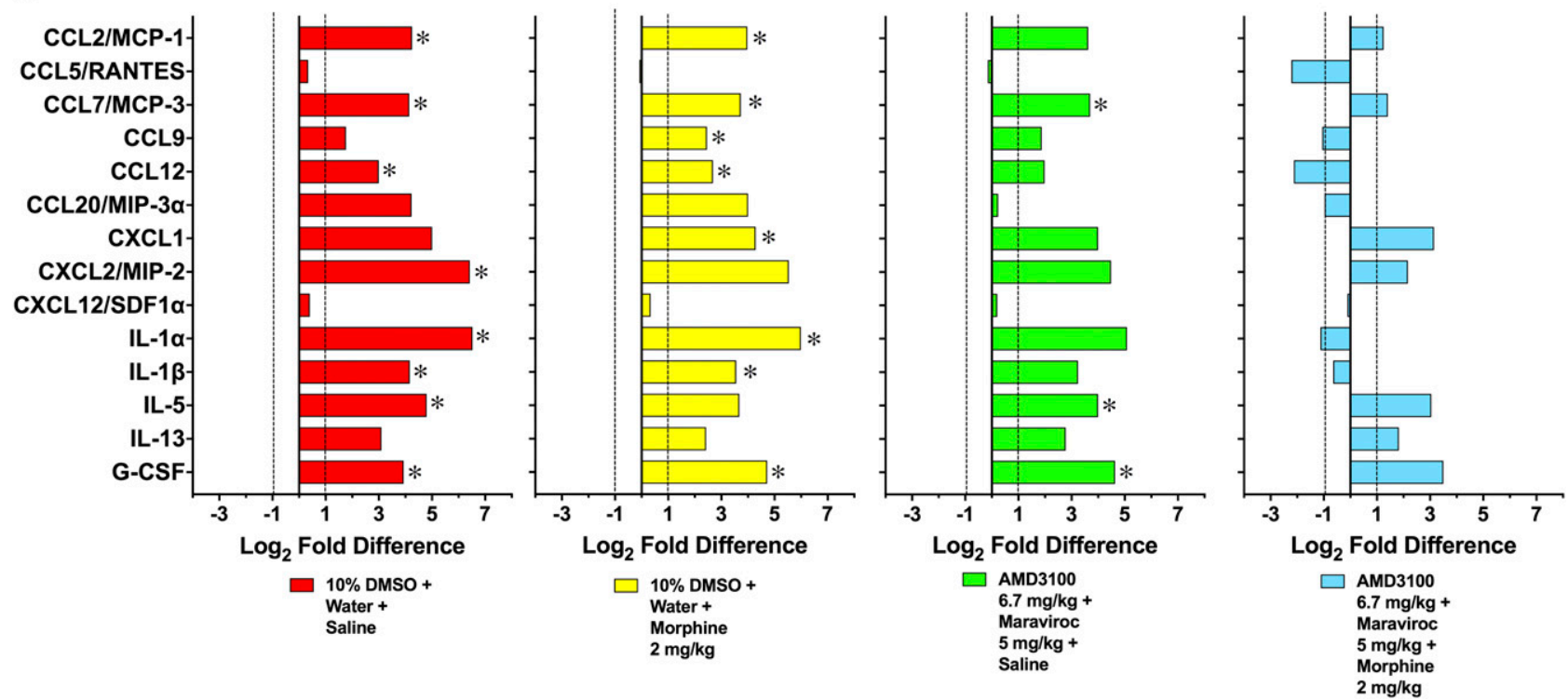

Fig. 8. Effect of incision alone and treatment postincision with morphine alone, AMD3100 + maraviroc, or morphine + AMD3100 + maraviroc on mRNA levels for a panel of chemokines and cytokines. The draining popliteal lymph node was harvested 1 hour postincision and was extracted and processed to obtain mRNA, which was analyzed using the $\mathrm{RT}^{2}$ Profiler Array (see Materials and Methods). (A) mRNA expression heat map of the popliteal lymph nodes of rats receiving an incision with and without treatments. Treatments are indicated at the bottom of each column. Color scale is a representation of the $\log _{2}$ fold change in mRNA levels of various immune mediators compared with untreated baseline lymph nodes, ranging from bright red (upregulated to over $\log _{2} 6$-fold) to bright green (downregulated to under $\log _{2} 2$-fold). Black represents 0 -fold change in mRNA expression. Data are presented as the mean level of mRNA expression in lymph nodes from three individual animals. (B) Fold change of mRNA expression in popliteal lymph nodes of rats receiving an incision with and without treatments. The data represent the $\log _{2}$ fold change in mRNA expression for a panel of immune mediators postincision, with or without treatments, compared with untreated, baseline popliteal lymph nodes. Dotted lines indicate limits of 2 -fold up- or downregulation from baseline. Data are presented as the mean level of mRNA expression in lymph nodes from three individual animals. ${ }^{2} P<0.05$ vs. baseline. 
G-CSF, were significantly increased 1 hour postsurgery. Morphine alone had a minimal effect on reducing the increased levels of mRNA for the aforementioned chemokines and cytokines. However, the combination of morphine with the two CRAs reduced the mRNA for these elevated inflammatory mediators to baseline levels. Clark et al. (2007) reported that incision induced expression of some cytokines (TNF- $\alpha$, IL-6, CXCL1/KC/IL-8, IL-1 $\beta$, and G-CSF) and neutrophil infiltration in paw tissues of mice following 30 minutes and 2 hours postsurgery, and that morphine reduced incisioninduced local cytokine expression. Additionally, Carreira et al. (2013) showed that CXCL1/KC signaling is important for recruiting neutrophils to the incision, and neutrophil infiltration is one of the mediators of postincisional pain in mice. Peters and Eisenach (2010) showed that intrathecal administration of an antibody specific for CCL2 24 hours postincision reversed mechanical allodynia in rats. In the experiments reported here, the results correlating the levels of mRNA for chemokines after incision and treatment with chemokine receptor inhibitors were unexpected. The cognate chemokines, CCL5/RANTES and CXCL12/SDF-1 $\alpha$, for maraviroc and AMD3100, respectively, were not upregulated by the incision. However, mRNAs for a panel of other unrelated chemokines and cytokines were upregulated, and the combination of morphine plus AMD3100 and maraviroc gave broad downregulation of these mRNAs. At present, we have no model to explain this finding.

Overall, for incisional pain, the evidence supports the conclusion that cytokines and chemokines are involved in the generation of pain stimuli. Since chemokines have also been shown to desensitize opioid receptors, blocking chemokine receptors in combination with opioids appears to be a novel and viable strategy in the field of pain treatment.

The results of the present studies show that maximal analgesia can be obtained by combining two chemokine receptor antagonists with subanalgesic doses of morphine. A strategy of pain relief that uses less morphine has many benefits. Among these are that it might diminish morphine's undesirable side effects. These include tolerance and dependence (Cowan and Macfarlane, 1975; Nozaki et al., 1975; Hasanein et al., 2015; Salmanzadeh et al., 2017), inhibition of gastrointestinal motility (Niwa et al., 2002; Gallantine and Meert, 2008), and respiratory depression (Emery et al., 2016; Whiteside et al., 2016). These preceding reports show that these adverse effects of morphine are dose dependent, and that the doses of morphine used in the present studies in combination with CRAs are sufficiently low that they would have the possibility of obviating the development of at least some of these adverse effects. Further testing of the effects of the CRAs in combination with morphine on undesirable side effects of the opioid is needed.

In conclusion, the addition of two CRAs, maraviroc and AMD3100 (having no analgesic activity themselves), to a subanalgesic dose of morphine significantly potentiated the analgesic activity of morphine to maximal levels, when tested on incisional pain in rats. The combination of two CRAs with morphine markedly reduced the dose of morphine required to obtain an equal analgesic effect. The results of the present study present an implementable strategy for reducing the dose of highly potent opioid analgesics, which could provide equivalent levels of analgesia and fewer and less serious side effects.

\section{Acknowledgments}

We thank Emily Dziedowiec for technical support.

\section{Authorship Contributions}

Participated in research design: Inan, Eisenstein, Chen, Tallarida, Geller, Meissler, Rawls, Cowan, Adler.

Conducted experiments: Inan, Watson, Doura, Meissler.

Performed data analysis: Tallarida, Inan.

Wrote or contributed the writing of the manuscript: Inan, Eisenstein, Geller, Meissler, Rawls, Cowan, Adler.

\section{References}

Akgün E, Javed MI, Lunzer MM, Powers MD, Sham YY, Watanabe Y, and Portoghese PS (2015) Inhibition of inflammatory and neuropathic pain by targeting a mu opioid receptor/chemokine receptor5 heteromer $\left(\mathrm{MOR}^{-\mathrm{CCR}_{5}}\right)$. $J$ Med Chem 58:8647-8657.

Ali H, Richardson RM, Haribabu B, and Snyderman R (1999) Chemoattractant receptor cross-desensitization. J Biol Chem 274:6027-6030.

Benamar K, Geller EB, and Adler MW (2008) Elevated level of the proinflammatory chemokine, RANTES/CCL5, in the periaqueductal grey causes hyperalgesia in rats. Eur J Pharmacol 592:93-95.

Bidlack JM (2000) Detection and function of opioid receptors on cells from the immune system. Clin Diagn Lab Immunol 7:719-723.

Brennan TJ, Vandermeulen EP, and Gebhart GF (1996) Characterization of a rat model of incisional pain. Pain 64:493-501.

Carreira EU, Carregaro V, Teixeira MM, Moriconi A, Aramini A, Verri WA Jr, Ferreira SH, Cunha FQ, and Cunha TM (2013) Neutrophils recruited by CXCR1/2 signalling mediate post-incisional pain. Eur J Pain 17:654-663.

Chen C, Li J, Bot G, Szabo I, Rogers TJ, and Liu-Chen LY (2004) Heterodimerization and cross-desensitization between the $\mu$-opioid receptor and the chemokine CCR5 receptor. Eur J Pharmacol 483:175-186.

Chen X, Geller EB, Rogers TJ, and Adler MW (2007a) Rapid heterologous desensitization of antinociceptive activity between mu or delta opioid receptors and chemokine receptors in rats. Drug Alcohol Depend 88:36-41.

Chen X, Geller EB, Rogers TJ, and Adler MW (2007b) The chemokine CX3CL1/fractalkine interferes with the antinociceptive effect induced by opioid agonists in the periaqueductal grey of rats. Brain Res 1153:52-57.

Chen X, Kirby LG, Palma J, Benamar K, Geller EB, Eisenstein TK, and Adler MW (2011) The effect of gp120 on morphine's antinociceptive and neurophysiological actions. Brain Behav Immun 25:1434-1443.

Chuang LF, Chuang TK, Killam KF Jr, Qiu Q, Wang XR, Lin JJ, Kung HF, Sheng W, Chao C, Yu L, et al. (1995) Expression of $\kappa$ opioid receptors in human and monkey lymphocytes. Biochem Biophys Res Commun 209:1003-1010.

Clark JD, Shi X, Li X, Qiao Y, Liang D, Angst MS, and Yeomans DC (2007) Morphine reduces local cytokine expression and neutrophil infiltration after incision. $\mathrm{Mol}$ Pain 3:28.

Cowan A and Macfarlane IR (1975) Effect of propranolol on antinociceptive, tolerance- and dependence-producing properties of morphine in rodents and monkeys. Eur J Pharmacol 34:87-94.

Dansereau MA, Gosselin RD, Pohl M, Pommier B, Mechighel P, Mauborgne A Rostene W, Kitabgi P, Beaudet N, Sarret P, et al. (2008) Spinal CCL2 pronociceptive action is no longer effective in CCR2 receptor antagonist-treated rats. $J$ Neurochem 106:757-769

Emery MJ, Groves CC, Kruse TN, Shi C, and Terman GW (2016) Ventilation and the response to hypercapnia after morphine in opioid-naive and opioid-tolerant rats. Anesthesiology 124:945-957.

Fleishaker DL, Garcia Meijide JA, Petrov A, Kohen MD, Wang X, Menon S, Stock TC, Mebus CA, Goodrich JM, Mayer HB, et al. (2012) Maraviroc, a chemokine receptor5 antagonist, fails to demonstrate efficacy in the treatment of patients with rheumatoid arthritis in a randomized, double-blind placebo-controlled trial. Arthritis Res Ther 14:R11.

Gallantine EL and Meert TF (2008) Antinociceptive and adverse effects of $\mu$ - and $\kappa$-opioid receptor agonists: a comparison of morphine and U50488-H. Basic Clin Pharmacol Toxicol 103:419-427.

Grimm MC, Ben-Baruch A, Taub DD, Howard OM, Resau JH, Wang JM, Ali H, Richardson R, Snyderman R, and Oppenheim JJ (1998) Opiates transdeactivate chemokine receptors: $\delta$ and $\mu$ opiate receptor-mediated heterologous desensitization. J Exp Med 188:317-325.

Guo CJ, Li Y, Tian S, Wang X, Douglas SD, and Ho WZ (2002) Morphine enhances HIV infection of human blood mononuclear phagocytes through modulation of $\beta$-chemokines and CCR5 receptor. J Investig Med 50:435-442.

Happel C, Steele AD, Finley MJ, Kutzler MA, and Rogers TJ (2008) DAMGO-induced expression of chemokines and chemokine receptors: the role of TGF- $\beta 1$. J Leukoc Biol 83:956-963.

Hasanein P, Teimuri Far M, and Emamjomeh A (2015) Salvia officinalis L. attenuates morphine analgesic tolerance and dependence in rats: possible analgesic and sedative mechanisms. Am J Drug Alcohol Abuse 41:405-413.

Heinisch S, Palma J, and Kirby LG (2011) Interactions between chemokine and mu-opioid receptors: anatomical findings and electrophysiological studies in the rat periaqueductal grey. Brain Behav Immun 25:360-372.

Huang W, Zheng W, Liu S, Zeng W, Levitt RC, Candiotti KA, Lubarsky DA, and Hao $\mathrm{S}$ (2014) HSV-mediated p55TNFSR reduces neuropathic pain induced by HIV gp120 in rats through CXCR4 activity. Gene Ther 21:328-336.

Kim CH and Broxmeyer HE (1999) Chemokines: signal lamps for trafficking of $\mathrm{T}$ and B cells for development and effector function. J Leukoc Biol 65:6-15.

Kwiatkowski K, Piotrowska A, Rojewska E, Makuch W, Jurga A, Slusarczyk J, Trojan E, Basta-Kaim A, and Mika J (2016) Beneficial properties of maraviroc on 
neuropathic pain development and opioid effectiveness in rats. Prog Neuropsychopharmacol Biol Psychiatry 64:68-78.

Lee YK, Choi DY, Jung YY, Yun YW, Lee BJ, Han SB, and Hong JT (2013) Decreased pain responses of $\mathrm{C}-\mathrm{C}$ chemokine receptor 5 knockout mice to chemical or inflammatory stimuli. Neuropharmacology 67:57-65.

Li N, Zhang L, Shu R, Ding L, Wang Z, Wang H, Yu Y, and Wang G (2016) Involvement of CCL3/CCR5 signaling in dorsal root ganglion in remifentanil-induced hyperalgesia in rats. Clin J Pain 32:702-710.

Luo X, Tai WL, Sun L, Pan Z, Xia Z, Chung SK, and Cheung CW (2016) Crosstalk between astrocytic CXCL12 and microglial CXCR4 contributes to the development of neuropathic pain. Mol Pain 12:1-15.

Mahajan SD, Schwartz SA, Shanahan TC, Chawda RP, and Nair MPN (2002) Morphine regulates gene expression of $\alpha$ - and $\beta$-chemokines and their receptors on astroglial cells via the opioid $\mu$ receptor. J Immunol 169:3589-3599.

Menichella DM, Abdelhak B, Ren D, Shum A, Frietag C, and Miller RJ (2014) CXCR4 chemokine receptor signaling mediates pain in diabetic neuropathy. Mol Pain 10 42.

Niwa T, Nakao M, Hoshi S, Yamada K, Inagaki K, Nishida M, and Nabeshima T (2002) Effect of dietary fiber on morphine-induced constipation in rats. Biosci Biotechnol Biochem 66:1233-1240.

Nozaki M, Akera T, Lee CY, and Brody TM (1975) The effects of age on the development of tolerance to and physical dependence on morphine in rats. $J$ Pharmacol Exp Ther 192:506-512.

Padi SS, Shi XQ, Zhao YQ, Ruff MR, Baichoo N, Pert CB, and Zhang J (2012) Attenuation of rodent neuropathic pain by an orally active peptide, RAP-103, which potently blocks CCR2- and CCR5-mediated monocyte chemotaxis and inflammation. Pain 153:95-106.

Peters CM and Eisenach JC (2010) Contribution of the chemokine (C-C motif) ligand 2 (CCL2) to mechanical hypersensitivity after surgical incision in rats. Anesthesiology 112:1250-1258.

Piotrowska A, Kwiatkowski K, Rojewska E, Makuch W, and Mika J (2016) Maraviroc reduces neuropathic pain through polarization of microglia and astrogliaevidence from in vivo and in vitro studies. Neuropharmacology 108:207-219.

Ragozzino D (2002) CXC chemokine receptors in the central nervous system: role in cerebellar neuromodulation and development. J Neurovirol 8:559-572.

Salmanzadeh H, Azizi H, and Semnanian S (2017) Adolescent chronic escalating morphine administration induces long lasting changes in tolerance and dependence to morphine in rats. Physiol Behav 174:191-196.

Szabo I, Chen XH, Xin L, Adler MW, Howard OMZ, Oppenheim JJ, and Rogers TJ (2002) Heterologous desensitization of opioid receptors by chemokines inhibits chemotaxis and enhances the perception of pain. Proc Natl Acad Sci USA 99: 10276-10281.

Tallarida CS, Bires K, Avershal J, Tallarida RJ, Seo S, and Rawls SM (2014) Ethanol and cocaine: environmental place conditioning, stereotypy, and synergism in planarians. Alcohol 48:579-586.

Tanaka T, Minami M, Nakagawa T, and Satoh M (2004) Enhanced production of monocyte chemoattractant protein-1 in the dorsal root ganglia in a rat model of neuropathic pain: possible involvement in the development of neuropathic pain. Neurosci Res 48:463-469.

Tran PB and Miller RJ (2003) Chemokine receptors: signposts to brain development and disease. Nat Rev Neurosci 4:444-455.

Wetzel MA, Steele AD, Eisenstein TK, Adler MW, Henderson EE, and Rogers TJ (2000) $\mu$-Opioid induction of monocyte chemoattractant protein-1, RANTES, and IFN- $\gamma$-inducible protein-10 expression in human peripheral blood mononuclear cells. J Immunol 165:6519-6524.

Whiteside GT, Hummel M, Boulet J, Beyenhof JD, Strenkowski B, John JD, Knappenberger T, Maselli H, and Koetzner L (2016) Robustness of arterial blood gas analysis for assessment of respiratory safety pharmacology in rats. J Pharmacol Toxicol Methods 78:32-41.

Wick MJ, Minnerath SR, Roy S, Ramakrishnan S, and Loh HH (1996) Differential expression of opioid receptor genes in human lymphoid cell lines and peripheral blood lymphocytes. J Neuroimmunol 64:29-36.

Xie F, Wang Y, Li X, Chao YC, and Yue Y (2016) Early repeated administration of CXCR4 antagonist AMD3100 dose-dependently improves neuropathic pain in rats after L5 spinal nerve ligation. Neurochem Res 41:2289-2299.

Zhang N, Hodge D, Rogers TJ, and Oppenheim JJ (2003) $\mathrm{Ca}^{2+}$-independent protein kinase Cs mediate heterologous desensitization of leukocyte chemokine receptors by opioid receptors. J Biol Chem 278:12729-12736.

Zhang N, Inan S, Cowan A, Sun R, Wang JM, Rogers TJ, Caterina M, and Oppenheim JJ (2005) A proinflammatory chemokine, CCL3, sensitizes the heat- and capsaicin-gated ion channel TRPV1 [published correction appears in Proc Natl Acad Sci USA (2005) 102:7050]. Proc Natl Acad Sci USA 102:4536-4541.

Zhang N, Rogers TJ, Caterina M, and Oppenheim JJ (2004) Proinflammatory chemokines, such as C-C chemokine ligand 3, desensitize mu-opioid receptors on dorsal root ganglia neurons. J Immunol 173:594-599.

Address correspondence to: Saadet Inan, Center for Substance Abuse Research, Lewis Katz School of Medicine at Temple University, 3500 North Broad Street, Philadelphia, PA 19140. E-mail: sinan@temple.edu 